\title{
ESTUDIO ICTIOLÓGICO EN EL EMBALSE DE JOAQUÍN COSTA (RÍO ÉSERA, HUESCA)
}

\author{
C. Granado-Lorencio, L. Encina, C. Escot-Muñoz, E. Mellado-Álvarez y A. Rodriguez-Ruiz. \\ Departamentode Biología Vegetal y Ecología. Facultad de Biología. Universidad de Sevilla. Apartado de corre- \\ os 1095,41080 , Sevilla.
}

\section{RESUMEN}

\begin{abstract}
Durante los años 1994 a 1996 se realizó el estudio de la ictiofauna del embalse de Joaquín Costa (Río Ésera, Huesca), comprendiendo el periodo de "equilibrio" colonización-gestión de la masa de agua (prevaciado) y los primeros estadios de la recolonización (post-vaciado). Se aplicaron técnicas de captura y de señales con ecosonda, con el objetivo de comparar las estimas con los datos del vaciado y realizar los ajustes del calibrado.

La asociación íctica estaba formada por carpa común, carpa de espejo, rutilo, black-bass, madrilla, barbo de Graells, trucha común, brema blanca y lucioperca. La familia Cyprinidae fue dominante ( $97.3 \%$ de las capturas totales), y de forma accesoria, centrárquidos, pércidos y salmónidos. Tras el vaciado se hizo menos diversa, con la desaparición de la mayoría de las especies exóticas y predominio de las nativas.

La aplicación de distintos métodos de análisis de la ecointegración arrojaron una estima total de las poblaciones entre 292575 y 68544 peces. Las maniobras de recogida de ejemplares durante el vaciado, aguas abajo, confirmó la estima obtenida a partir de la agrupación de transectos y estaciones de muestreo.
\end{abstract}

Palabras clave: asociación íctica, estilos de vida, ecosonda, poblaciones, colonización

\section{ABSTRACT}

The stud,: of the ictiofauna of Joaquin Costu reservoir (Ésera River, Huesca) was carried outfrorn 1994 to 1996. The study covered two periods of time; the pre-emptying phase characterised by fish colonisation and water management, and the post-emptying when fishes recolonise the reservoir. Results ohtuined with net and ecosounding methods were compured with direct fish estimutions during the flushing in order to validate the procedure followed in the study.

The fish fauna assemblage was formed by common curp, mirror curp, roach, black-bass, nase, barbel of Graells, common trout, white breurn and pikeperch. The family Cypriniúue was dominunt (97.3\% of the totul captures), followed by centrarquids, percids and salmonids. Thejish assemhluge became less diverse, after the flushing with the disappearunce of most of the exotic species and prevalence of the native ones.

The different methods of ecointegration provided u total estimution that rungesfroin 292575 to 68544 fishes. The recovering of individuals during the flushing confirms the comhined results obtuinedfrom the echosounding transects and sampling stations methods.

Keywords: fish assemblage, life styles, echosounding, fish population, settlement.

\section{INTRODUCCIÓN}

El estudio ecológico de la ictiofauna de los embalses tiene un elevado interés científico, debido a que constituye un marco natural de referencia para conocer las adaptaciones desarrolladas por las especies, residentes en el río, una vez colonizan estos ecosistemas artificiales. Este tipo de proyectos constituyen una actividad bastante habitual en otros países (Merle et al., 1994: Duncan y Kubêcka, 1995): aunque no así en España (Sancho y Granado Lorencio, 1988).
Los procesos de colonización y la capacidad de dispersión de las especies fluviales van a determinar la evolución de las asociaciones, a lo largo del tiempo, en un mecanismo de interacción entre el fenotipo de las especies y el comportamiento de la masa de agua (Douglas y Matthews, 1992).

La oportunidad de realizar el seguimiento a un embalse y su ictiofauna que, por razones técnicas, iba a ser vaciado varias veces, constituía un atractivo adicional a lo anteriormente expuesto: permitiendo conocer tanto los sucesivos episodios 
de colonización de la masa de agua, como la posibilidad de comprobar el grado de precisión de las metodologías habitualmente utilizadas. Ibamos a participar de un inmenso experimento, pocas veces realizado.

Los periodos temporales considerados corresponden a la fase anterior al primer vaciado (equilibrio con el manejo tradicional del embalse) y entre dos vaciados sucesivos, Los objetivos, al inicio del estudio, en cada una de las fases consideradas, se pueden clasificar en cuatro grupos:

1. Conocer la composición cualitativa de las asociaciones piscícolas.

2. Conocer ciertos aspectos relativos a las estrategias de vida de las especies, antes del vaciado (1994-1995).

3. Comprobar y ajustar los diferentes métodos de estima de la abundancia y biomasa íctica.

4. Estudiar los efectos derivados del vaciado sobre las poblaciones de peces.

\section{METODOLOGÍA}

Inicialmente, se realizaron ensayos sobre distintos diseños de muestreo, tanto con redes como con sistemas de ecointegración, a fin de alcanzar un modelo representativo del embalse; y que las estimas obtenidas pudieran ser contrastadas durante las maniobras de vaciado. Las técnicas de muestreo establecidas se encuentran descritas en Granado-Lorencio et al. (1996). Para conocer la composición cualitativa y la estructura en talla de la comunidad íctica se emplearon artes de pesca pasivas (trasmallos y nasas), utilizándose técnicas de ecointegración para estimar la abundancia y la distribución por tamaño (Fig. 1). La comparación de ambos resultados permitieron conocer el número y la biomasa íctica a lo largo de varias campañas de muestreo (Fig. 2).

La determinación de las edades se realizó mediante el estudio de las escamas; y los modelos de

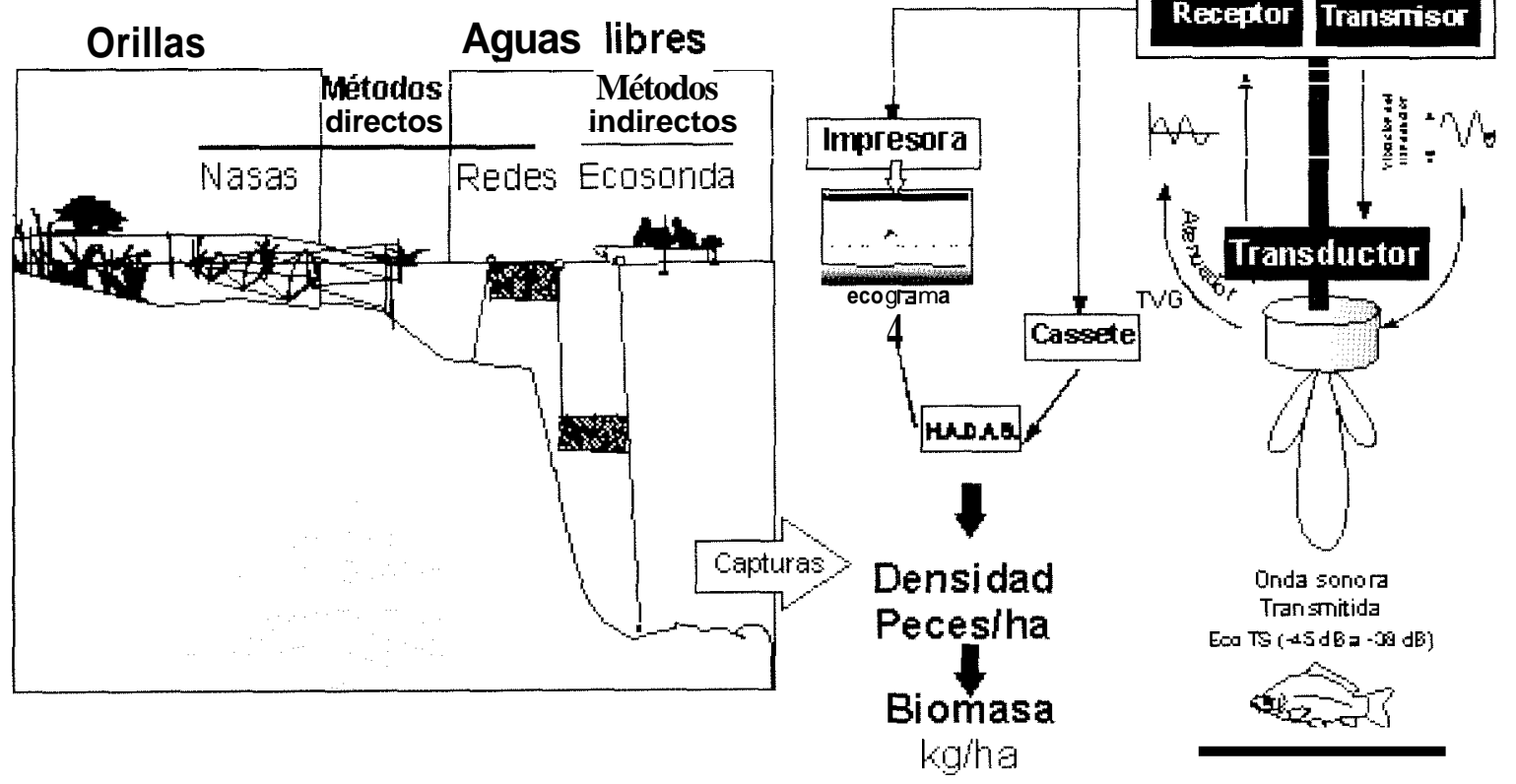

Figura 1. Diseño general de muestreo: Métodos directos (artes de pesca) e indirectos (Ecointegración). General sampling design: Direct methods (fishing nets) and indirect (Ecointegration). 
crecimiento se construyeron a partir de la función de Von-Bertalanffy. Se analizó el estado reproductivo mediante el análisis del desarrollo gonadal (peso total de la gónada y tamaño del huevo) y se evaluó el sex-ratio para cada especie. Para la alimentación se analizaron los contenidos gastrointestinales (frecuencia-ocurrencia y abundancia). Las técnicas de estudio se describen en Granado-Lorencio (1996).

\section{ICTIOFAUNA}

Las especies capturadas en el embalse (Fig. 3) fueron Cyprinus carpio (carpa común), Cyprinus carpio var. specularis (carpa de espejo), Rutilus rutilus (rutilo), Micropterus salmoides (blackbass), Chondrostoma toxostoma miegii (madri1la), Barbus graellsi (barbo de Graells), Salmo truttu fario (trucha común), Blicca bjöerkna (brema blanca) y Stizostedium lucioperca (lucioperca).

La ictiofauna durante 1994 y 1995 (periodo anterior al primer vaciado) pertenecen a cuatro familias, siendo la más representada la de los ciprínidos con un $97.3 \%$ de ejemplares capturados; seguida de los centrárquidos $(1.7 \%)$, pércidos y salmónidos con sólo un $0.6 \%$ y $0.4 \%$ respectivamente (Fig. 4). Dentro de la familia mayoritaria (Cyprinidae) se han capturado 6 especies, mientras que las demás sólo aportaron una. Existen un total de tres especies nativas (barbo de Graells, madrilla y trucha común) coexistiendo con seis especies exóticas. La riqueza piscícola del embalse es bastante alta, no habitual para este tipo de embalse (regadío) y en la comunidad fluvial que se anega, como ha sido demostrado en otras regiones geográficas (Fernando y Holçik, 1991). El

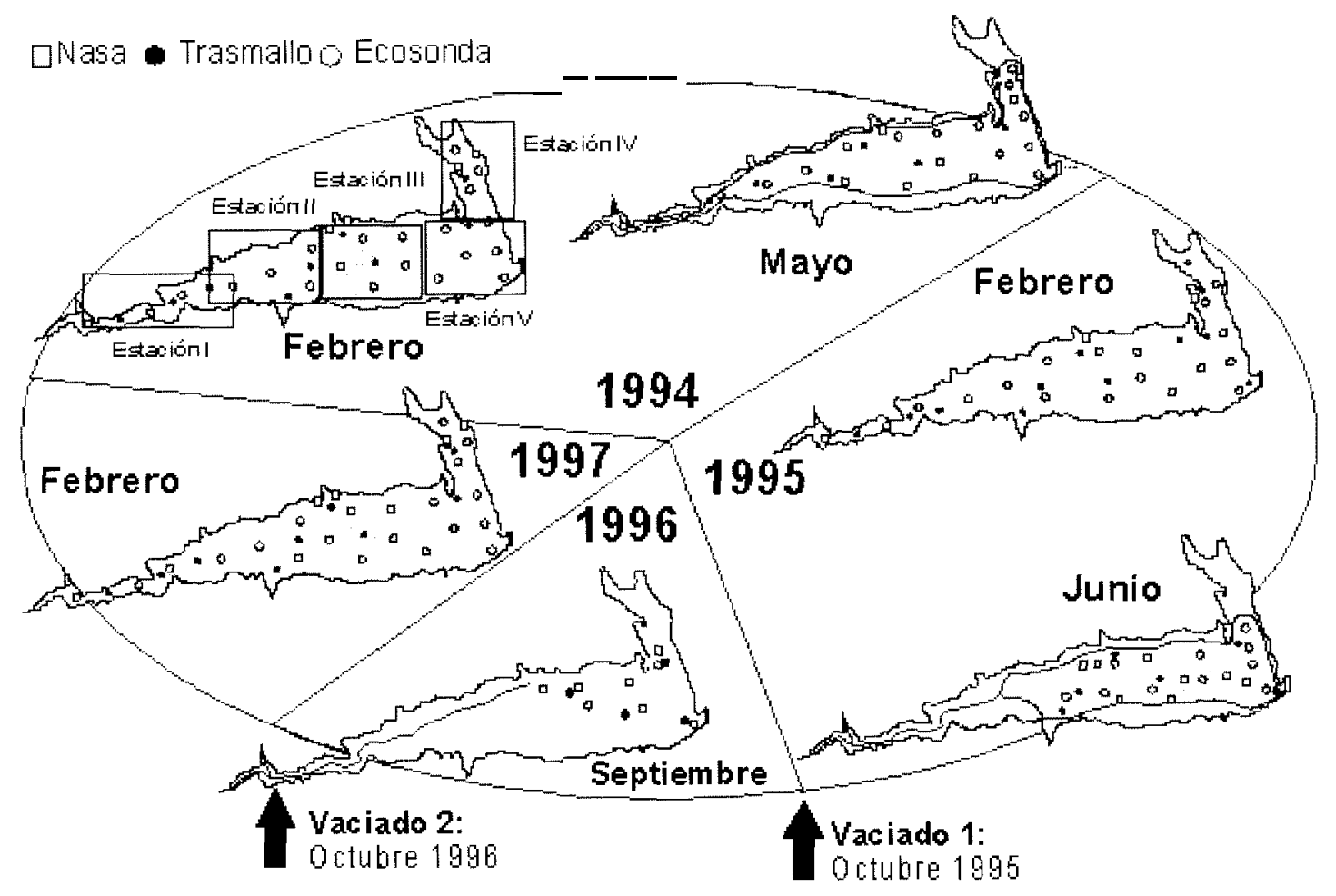

Figura 2. Campañas y distribución de las estaciones de muestreo. Sampling periods and distribution of the sampled stations 
predominio de los ciprínidos corresponde a un conjunto de fenómenos de supervivencia, característicos de las especies de ciclo corto, con gran capacidad pionera (Penczack, 1995) y estrategias reproductoras poco exigentes (Kubècka, 1992).

Durante 1994, las especies dominantes en la asociación íctica del embalse fueron barbo de Graells $(36 \%)$, madrilla $(25.3 \%)$ y rutilo $(23.4$ $\%)$; las dos primeras aportan el $61.3 \%$; representando las tres especies el $84.7 \%$ del total. Otras especies aparecieron de manera errática. Sin embargo, en el año 1995 las que aportaron un mayor porcentaje fueron las dos primeras (madri1la, $32.0 \%$ y barbo de Graells, 27.3\%); siendo el porcentaje conjunto del $59.3 \%$. El rutilo descendió su presencia al $13.6 \%$ (siendo el $72.9 \%$ el porcentaje de las tres juntas). La brema blanca no apareció durante las capturas de 1994, pero alcanzó el $14 \%$ en el siguiente año. Especies como black-bass, carpa de espejo y carpa común mantuvieron porcentajes similares en ambos periodos de estudio.

\section{ESTILOS DE VIDA}

Excepto en madrilla y rutilo la estructura de tallas en las poblaciones se caracteriza por la ausencia de juveniles y dominancia de ciertos rangos de talla; en concreto las correspondientes a las edades 6 y 7 en el caso del barbo, y a ejemplares, en general, de más de 9 años en las carpas (Fig. 5). Sólo en la carpa común, carpa de espejo y black-bass la estructura de edad de las capturas no sufrieron cambios significativos a lo largo de la diferentes campañas de muestro.

El análisis de los modelos de crecimiento aplicados a las distintas especies de ciprínidos, muestra el dimorfismo sexual en crecimiento propio de estas especies. A una misma edad las hembras son más grandes que los machos. Además las longitudes máximas que predicen los modelos corresponden a las esperadas cuando las especies crecen en sistemas leníticos como son los embalses, es decir crecimientos mucho mayores que en los ríos (Granado-Lorencio et al., 1996).

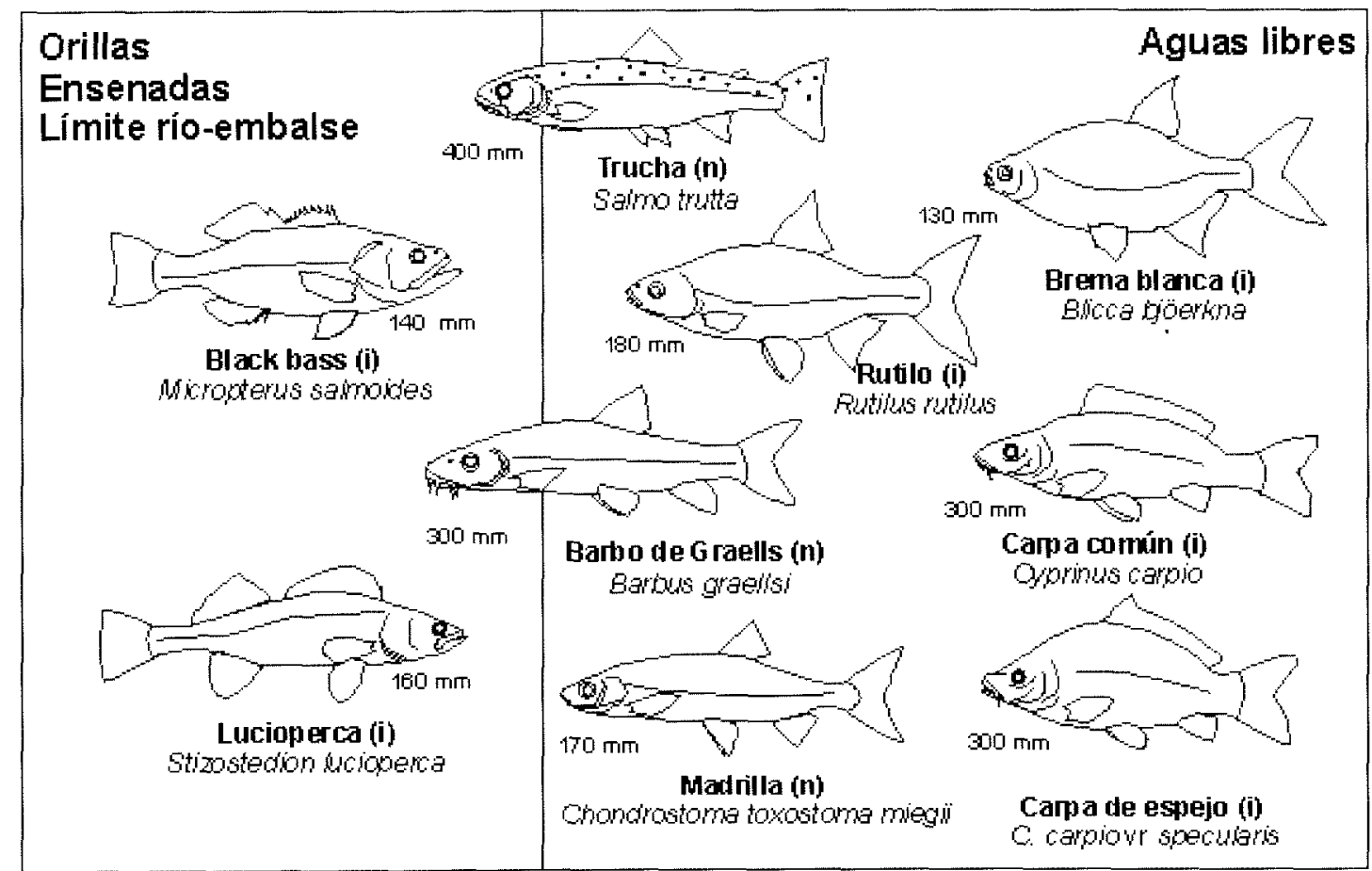

Figura 3. Ictiofauna del Embalse de Barosana. Especie introducida (i); nativa (n); en milímetros longitud total de captura. Fish fauna of the Barasona Reservoir. (i) Introduced species: $(n)$ native, in millimeters total length of captured specimens. 
En el total de capturas se registró un numero mayor de hembras que de machos para barbos, madrillas y rutilos, mientras que no existieron diferencias en la proporción de sexos en las bremas y en los dos tipos de carpas.

El desarrollo de las gónadas en los ejemplares capturados (peso total de la gónada y tamaño del huevo) se adecuó a su ciclo reproductor. En el caso de los ciprínidos los máximos desarrollos gonadales corresponden al periodo comprendido entre Febrero y Junio, que incluyen las campañas realizadas en el año 1994 y 1995.

\section{ALIMENTACIÓN}

Podemos agrupar las especies bajo estudio en dos grupos desde el punto de vista de la amplitud de su nicho trófico. El primer grupo estaría constituido por aquellas especies con carácter más eurífago, es decir, con mayor amplitud del nicho: Pertenecen a este grupo los barbos, las carpas y la brema. Las restantes especies, madrilla, rutilo, trucha y black bass, estarían incluidas dentro de las especies con un carácter más estenófago. En cualquier caso, la amplitud del nicho trófico registró cambios estacionales, especialmente evidentes en barbo y carpa de espejo. En la primera de las especies la diversidad trófica descendió desde invierno a verano en los dos años de estudio. En la carpa de espejo este cambio se observó igualmente en el primer año de estudio, pero no así en el segundo. Resulta también importante destacar el descenso progresivo registrado a lo largo de los dos años de estudio (desde la primera a la cuarta campaña) en la diversidad trófica de ambas especies. Los cambios estacionales registrados en las restantes especies no son tan acusados como en las dos primeras.

Desde el punto de vista de la composición cualitativa de la dieta, podemos diferenciar cuatro grupos; trucha y black bass, depredadores sobre macroinvertebrados; rutilo y brema, con predominio de crustáceos planctónicos; boga, detritívora; y barbo y carpas, con alternancia de explotación sobre elementos tanto del plancton como del bentos (Fig. 6).

Comparando la similaridad y el solapamiento trófico de la dieta de cada una de las especies, evaluadas para el conjunto de todo el ciclo anual, podemos diferenciar tres grupos de especies: las

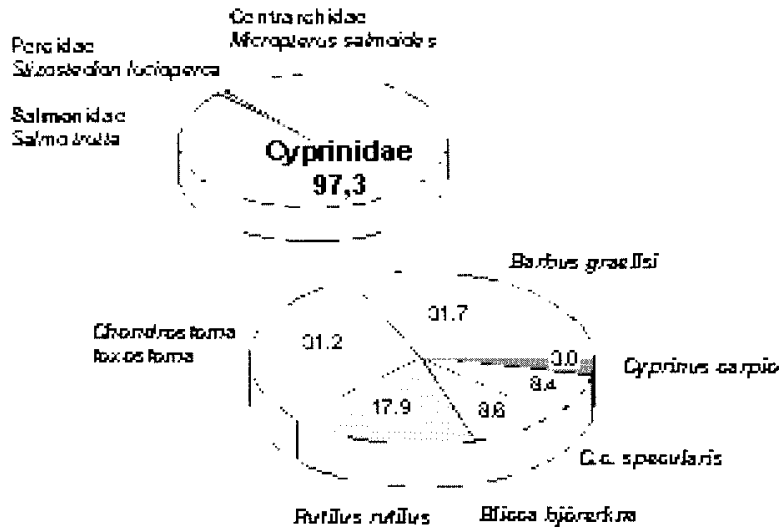

Figura 4. Abundancia relativa de las especies ícticas capturadas durante 1994-1995. Relative abundance of the fish species captured during 1994-1995.

que presentan índices de similaridad y solapamiento altos en ambos casos (barbo y carpas, tanto común como de espejo), aquellas con índices de similaridad moderado o bajo, pero alto de solapamiento (brema y rutilo), y por último, las que registran valores bajos para ambos índices (madrilla, trucha y black bass).

\section{POBLACIONES}

Las estimas de abundancia íctica obtenidas en Febrero de 1994, con un volumen del embalse de $70 \mathrm{hm}^{3}$, oscilan entre 22980 peces y 175000 peces según los diferentes métodos ensayados,. En Mayo de $1994(64 \mathrm{hm}$ ') las estimas oscilaron entre 45952 y 192000 peces. Si consideramos el periodo comprendido entre ambas campañas, abarcando un tiempo de generación de las poblaciones, la estima media en cada uno de íos métodos utilizados nos da unos efectivos poblacionales comprendidos entre 34466 y 183500 peces.

Considerando el siguiente periodo (1995) las estimas obtenidas durante el mes de Febrero con un volumen del embalse similar al periodo anterior de muestreo $\left(79 \mathrm{hm}^{3}\right)$, osciló entre $98000 \mathrm{y}$ 381950 peces. La evaluación de las poblaciones durante el mes de Junio del mismo año $\left(43 \mathrm{hm}^{3}\right.$, valor mínimo alcanzado del agua embalsada 


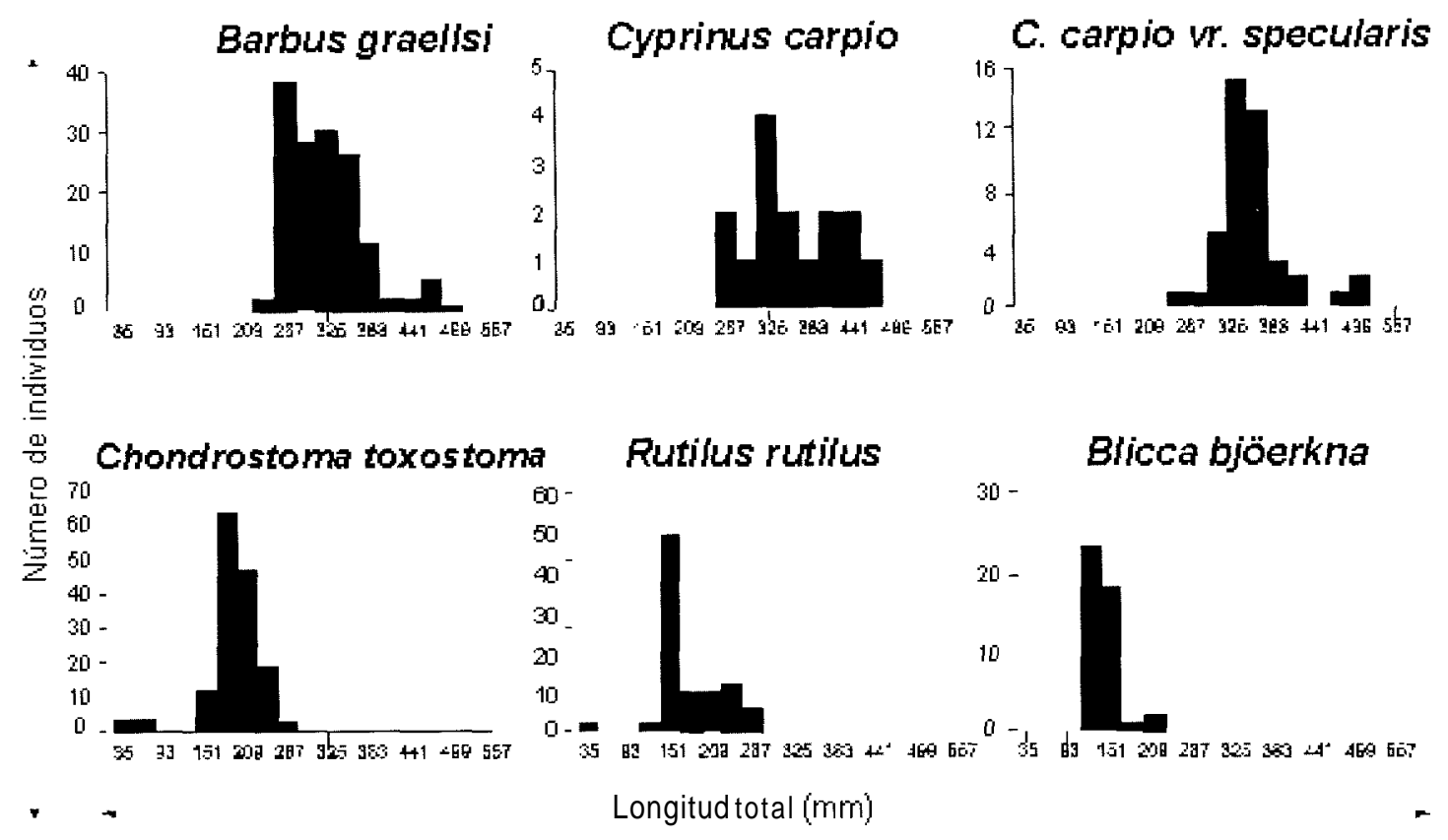

Figura 5. Estructura de talla de las especies pertenecientes al grupo de los ciprínidos. Size structure of the cyprinids species

durante este estudio), arroja una cifra comprendida entre 73100 y 203200 peces. Esta estima que se ve modificada cuando el análisis se realiza con las medias obtenidas durante las dos campañas de 199.5, que corresponden con el máximo y el mínimo volumen de agua embalsada. Los valores calculados se encontraron en el rango de $855.50 \mathrm{y}$ 292575 peces. La mayor densidad de peces se sitúa en las zonas de menor profundidad (orillas y ensenadas). La máxima en Febrero de 1995 y la mínima en Febrero de 1994 (asociadas a zonas de máxima profundidad).

En ambos periodos de estudio (1994 y 1995) las biomasas estimadas para las campañas invernales (Febrero) fueron mayores que las obtenidas en primavera-verano, independientemente del método empleado en la estima de densidades (a excepción del método cuarto). En general las biomasas estimadas en 199.5 fueron superiores a las de 1994, evidenciando un proceso expansivo de las poblaciones. Esta variabilidad en la biomasa íctica es característica de embalses con fuertes oscilaciones de nivel (Aggus y Lewis, 1977). Es difícil enmarcar estas variaciones en la biomasa total del embalse en la presencia de nuevas especies exóticas, como demostró De Silva (1988).

\section{VACIADO DEL EMBALSE}

Durante los días del vaciado, realizado en octubre de 199.5, se evaluaron por parte del personal asignado por Confederación Hidrográfica del Ebro, el total de peces que salieron por los túneles de fondo. Se establecieron un total de tres zonas de toma de muestras: tramo del río desde el pie de presa hasta las balsas de oxigenación, en las propias balsas y en el tramo de confluencia con el río Cinca. La base de las estimas fueron los recipientes de traslado de peces muertos (primera zona), las pescas eléctricas en las balsas de recuperación de ictiofauna viva por el personal de Confederación y el muestreo visual.

En una muestra, elegida al azar, de 43 kilogramos de peces recogidos, se clasificaron las especies, tras lo cual se midieron los ejemplares $(\mathrm{mm})$ y se pesaron $(\mathrm{g})$. Las especies que aparecieron fueron las mismas encontradas en el muestreo del 
embalse, a excepción de Leuciscus cephalus (bagre) que aparece por primera vez en el vaciado. Los porcentajes de aparición estimados en ambos casos (muestreo del embalse vs vaciado), no obstante, son en algunas especies diferentes. Las especies de aparición rara mantienen su presencia. No así ocurre con algunas de frecuencia superior al $10 \%$. Mientras que los barbos y rutilos mantienen una importancia similar, carpa de espejo se infravalora en el muestreo con redes y ocurre lo contrario en brema y madrilla, con mayores porcentajes a los obtenidos durante el vaciado.

Estos resultados parecen expresar que este tipo de artes de pesca da buena información sobre la composición cualitativa de la comunidad de peces, pero presentan algunas imprecisiones sobre su importancia cuantitativa, en especial respecto a las especies más abundantes, y no así con las raras. No obstante, existe la posibilidad de que la muestra utilizada no fuera suficientemente representativa, y que se produzca una salida diferenciada de las especies durante el vaciado, primero unas especies y después mayoritariamente otras; o incluso que las madrillas, con marcada reofilia positiva (Rodríguez-Ruiz y GranadoLorencio, 1992), remontasen los tributarios cuando comenzó el desagüe. Que las especies de pequeño tamaño quedaran envueltas en la lengua de barro que salió del embalse, y por ende fueran difíciles de contabilizar hasta pasados bastantes días después del vaciado, resulta posible, pero no ha sido valorada cuantitativamente.

Algunas de las especies que aparecen en la estadística del vaciado, formarían parte de las poblaciones del río, ya que no suelen formar parte de la ictiofauna de los embalses, quedando confinadas en las zonas de contacto embalse-tributario (c.f. Kapetsky y Petr, 1984), caso de gobio, Gobio gobio; bagre, Leuciscus cephalus y lobo de río, Noemacheilus barbatulus. A partir de esta muestra se aplicó elfactor de ampliación de

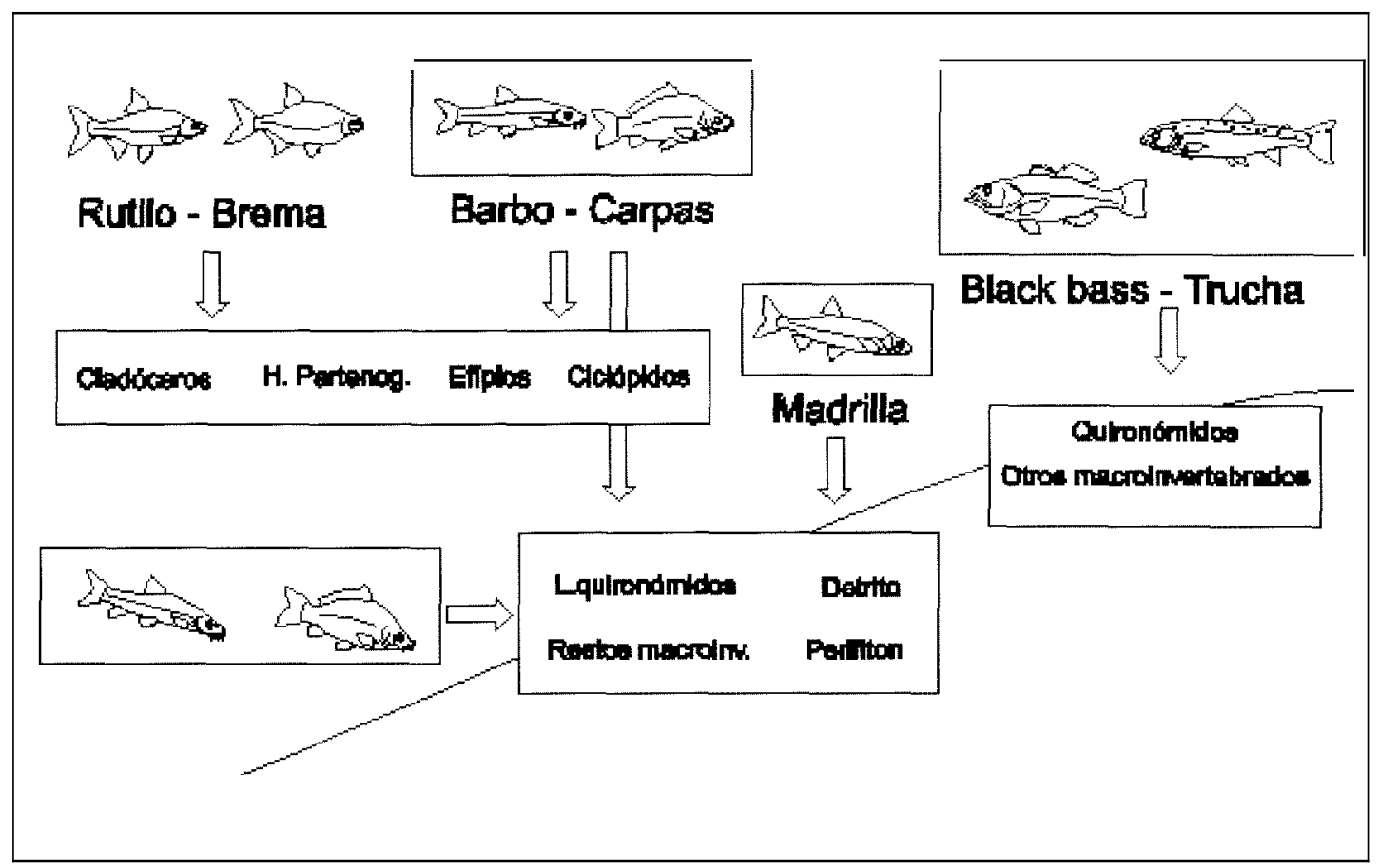

Figura 6. Estrategia alimenticia de las especies ícticas más representativas del embalse. Feeding strategy of the species more representative of the Barasona fish fauna. 


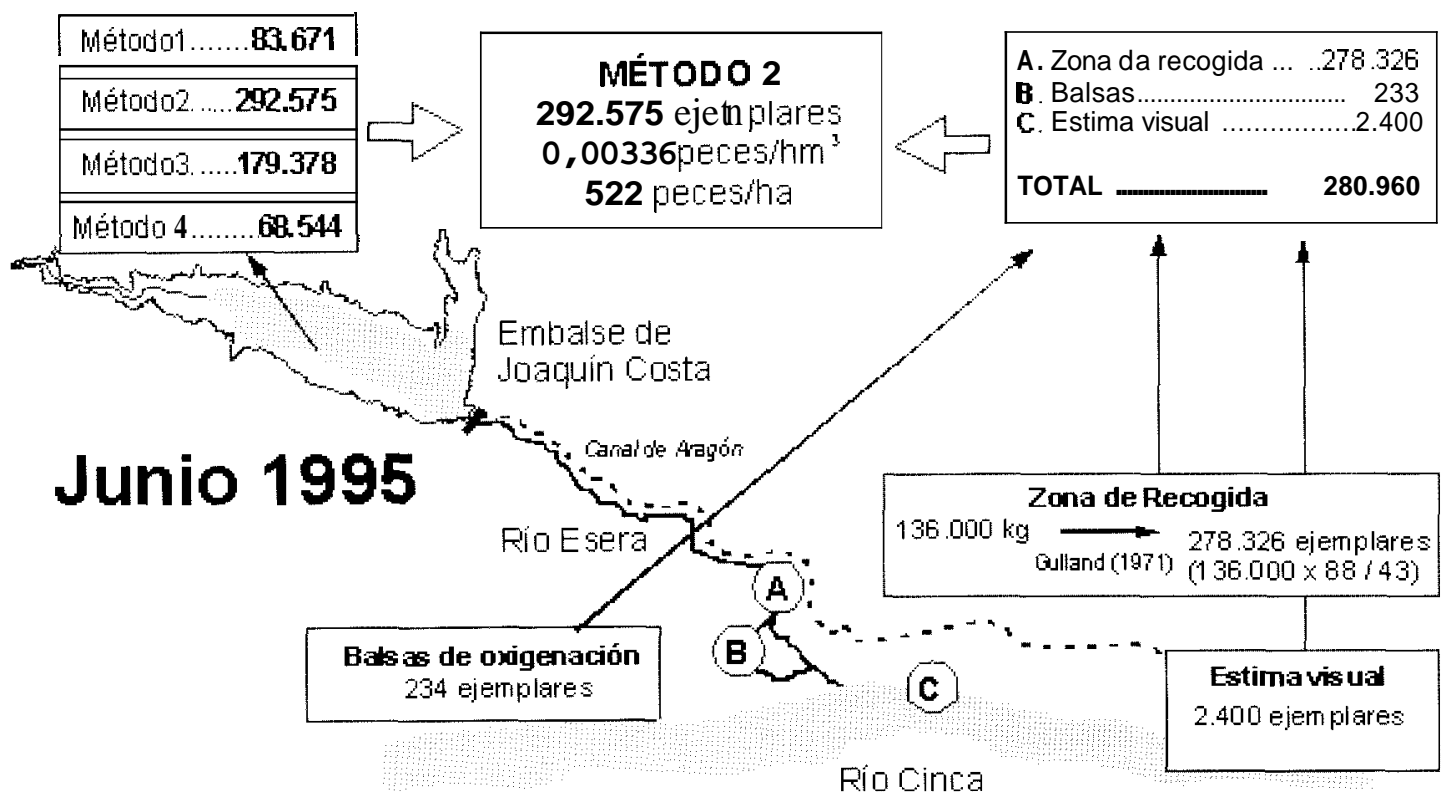

Octubre 1995

Figura 7. Estadística pesquera del primer vaciado del embalse. Fishing statistic of the first casting of the reservoir.

Gulland para estimar los efectivos poblacionales. Si el total de biomasa de peces recogidos en la maniobra de vaciado (alrededor de 136000 kilogramos) y la muestra analizada fue de 43 kilogramos y 88 peces, dicha extrapolación nos da un número estimativo de peces que han salido del embalse de 278 326. A este valor se les sumó los aparecidos en las balsas de oxigenación (234) y los observados en el muestreo visual (2400), dando un total de peces de 280960.

Como se observa en la figura 7, de los diferentes métodos aplicados para la estima de la abundancia íctica mediante los muestreos con ecosonda, el método consistente en agrupar los transectos usando estaciones de muestreo y extrapolando al total de volumen del embalse (Método 2), es el que proporcionó la estima más cercana al nútnero total de peces obtenidos durante las labores del vaciado. Este grado de coincidencia no debe utilizarse en sentido absoluto. Si bien parece que en el vaciado del embalse de Joaquín Costa la mayoría de los peces salieron por los desagües, experiencias realizadas en Francia estiman que entre el 15 y $20 \%$ de los peces se quedan atrapados en el lodazal del embalse o remontan aguas arriba los tributarios del embalse, en un comportamiento típicamente reófilo. Esta posibilidad no ha sido evaluada en nuestro caso, lo que podría alejar ambas estimas. De igual modo, la dificultad que implica contabilizar la totalidad de peces arrastrados por el vaciado, debido a que muchos de ellos quedan inmersos en la lengua de lodo, atrapados en la vegetación de orilla del río o difícilmente visibles por el personal asignado a estas labores por cansancio, durante las varias horas que dura la maniobra, el recuento del total de peces que salieron del embalse está sometido a un cierto error.

A pesar del grado de coincidencia en las estimas de abundancia entre el método 2 y el vaciado, existe cierto grado de disparidad entre los resultados de biomasa. Esta mayor diferencia posiblemente sea debida a una sobrestima de la biomasa íctica durante el vaciado, quizás originado por la aplicación de un método de estima poco riguroso. Esto determina futuras líneas de investigación, aprovechando la utilidad de las tareas asociadas al vaciado de embalses en el desarrollo 
de métodos de estimas ícticas cada vez más precisos y depurados, que permitirán una adecuada gestión de nuestras especies ícticas epicontinentales.

\section{COLONIZACIÓN}

En septiembre de 1996, tras el primer vaciado del embalse, se llevó a cabo una campaña para evaluar el efecto del mismo, y la colonización de las especies. En total fueron capturadas cinco especies, riqueza inferior a la observada en periodos anuales precedentes, si bien el rendimiento pesquero fue superior (437 ejemplares). Este resultado pone de manifiesto la reducción dramática de la riqueza existente en el embalse tras el vaciado (Fig. 8). Desaparecieron rutilo Rutilus rutilus, black-bass Micropterus salmoides, brema Blicca bjoerkna y lucioperca Stizostedium lucioperca; todas ellas con carácter de especies exóticas para la Península Ibérica, y por lo tanto íntimamente ligadas a Proyectos de repoblación con fines de pesca deportiva, con individuos provenientes de piscifactoría. Este proceso no se cumple con otras especies igualmente introducidas, como son las dos formas de carpas existentes, Cyprinus carpio y C.c. var. specularis. Los datos de que se disponen no permiten verificar si los ejemplares capturados (el 100\% de talla inferior a $200 \mathrm{~mm}$, no capturados con anterioridad en el embalse) pertenecen a reintroducciones (no existe constancia oficial), o si provendrían de reproducciones exitosas durante el periodo entre los dos vaciados.

El mismo proceso ha acontecido con relación a los barbos y madrillas, aunque con una explicación más natural (recolonización del embalse desde el río). En el caso de los barbos, el $100 \%$ de los ejemplares menores de $200 \mathrm{~mm}$ se capturan en 1996, antes nunca se capturaron; y siempre asociados a los hábitats someros y de orilla. Las madrillas con tallas pequeñas fueron capturadas durante este periodo, si bien esta especie también lo fue durante los años anteriores. Ambas especies remontan los ríos para reproducirse y quedan las formas juveniles en las áreas de freza, mientras que los adultos, tras la reproducción vuelven al embalse. El vaciado del mismo determina la pérdida de adultos o la migración aguas arriba, y la posterior colonización de una nueva masa de agua tras el vaciado. Estos patrones de recolonización se realizan espacialmente, así durante la fase inicial ocupan las zonas de orilla, para posteriormente desplazarse a las zonas más profundas, debido a las características morfométricüs del embalse de Barasona.

Las estimas obtenidas con la ecosonda muestran un total de peces del embalse similar al año anterior del vaciado, en la horquilla de 250000 a 300000 peces (Fig. 9). Estos resultados parecen indicar, en términos relativos (número), una determinada capacidad de recolonización. motivada por los focos de dispersión de los ríos Esera e Isábena (sin contemplar las posibles introducciones). Las poblaciones del río anualmente realizan colonizaciones del embalse, de forma más o menos explosiva. Las especies potamodromas como barbo de Graells y madrilla se reproducen en el río y descienden, los adultos, hacia sectores más bajos, en este caso el embalse. Cuando la masa de agua está sometida a fluctuaciones estacionales por el Programa de Regadíos, son mayoritariamente los adultos los que ocupan el embalse, quedando las formas larvarias y juveniles en los tramos altos. Si el vaciado es total, el embalse ocupado por efectivos poblacionales de todas las cohortes, siempre que no existan fenómenos competitivos (primeras etapas de colonización de un nuevo ecosistema-fase expansiva). A esta fase, en años siguientes, le irán sucediendo etapas de colonización que vendrán moduladas por íos ritmos de utilización del agua con fines de regadío, llenado a cota máxima, descenso, etc. Nunca se alcanzarán etapas de madurez (Perturbación intermitente) aunque sí de 'equilibrio' con su manejo (características descritas en los años 1994 y 1995). Cuantas veces se vacíe el embalse, por motivos técnicos como los acontecidos en estos dos últimos años, de nuevo se iniciará una nueva etapa pionera.

En cuanto a la biomasa la discusión se modifica. Para evaluar la situación de la asociación pesquera del embalse no importa tanto el número como la biomasa. Las estimas de biomasa total para el año 1994, 1995 y tras el vaciado varían, siendo notablemente inferior tras este último. Tales resultados permiten discutir el compartimento de los peces en el embalse de Barasona, tanto en los periodos de perturbación intermitente, debidos al regadío, como en la fase de colonización de una nueva masa de agua. Durante 1994 


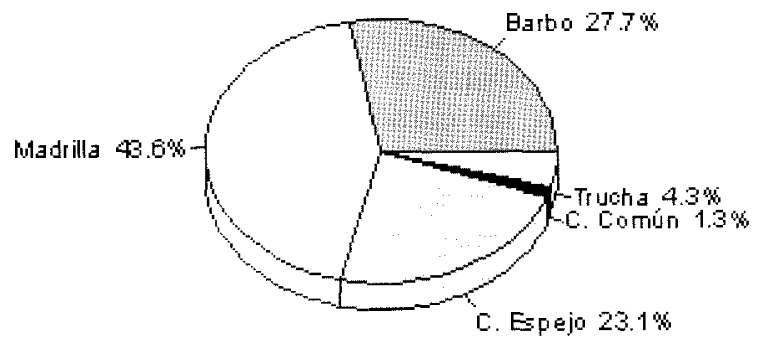

Figura 8. Abundancia relativa de las especies ícticas capturadas tras el primer vaciado. Relative abundance of the fish species captured after thr first casting.

y 1995, las biomasas de peces son similares, no alcanzándose diferencias significativas interanuales. Ello pudiera indicar un proceso de ocupación expansiva de las poblaciones de peces en un embalse de estas características (ajuste manejocolonización reiterada). La biomasa calculada para el periodo tras el vaciado pone de manifiesto la etapa pionera de colonización, con un valor cercano al $13.95 \%$ de la biomasa observada cuando el embalse está sometido a un régimen de explotación tradicional. El embalse se encontraba pues, antes del segundo vaciado, en un estado de expansión de las poblaciones nativas del río.

En Febrero de 1997, tras el segundo vaciado, se llevó a cabo una campaña de muestreo para evaluar las consecuencias del mismo. Los resultados obtenidos indican el drástico efecto que este segundo vaciado ha tenido sobre la ictiofauna, cuando aún las poblaciones estaban recuperándose del primer vaciado. Así, es significativo la prácticamente ausencia de capturas en las artes de pesca, a un mismo esfuerzo empleado. La abundancia íctica estimada con la ecosonda fue en esta ocasión de 162722 peces, lo que supone un brusco descenso de los efectivos poblacionales respecto al último muestreo, realizado durante el periodo entre los dos vaciados.

La secuencia temporal de los resultados obtenidos, a lo largo del periodo de estudio, evidencia una fase de expansión de las poblaciones residentes en la masa de agua del embalse, reforzadas por las fluviales que entran al mismo, una vez finalizado el periodo de regadíos. Se podría asimilar, este comportamiento, a una sustitución continua de fases de expansión (llenado) y de repliegue (regadíos), acorde con el manejo del embalse (c.f. Jenfins, 1970). Las especies que forman parte de la asociación del embalse atienden a procesos no determinantes (GranadoLorencio, 1991). Los niveles más bajos del volumen del embalse beneficiarían a las especies depredadoras (Aggus, 1979); mientras que su aumento favorece a las oportunistas que explotan organismos terrestres y macrófagos aéreos. La capacidad de carga del sistema nunca se alcanza.

Tras el vaciado, la ocupación de la nueva masa de agua se realiza a partir de las poblaciones residuales atrapadas en el lodazal del vaso y de las que proceden de los ríos que entran al embalse. La historia de la ictiofauna del embalse de Joaquín Costa empieza de nuevo.

\section{BIBLIOGRAFÍA}

AGGUS, L.R. 1979. Effects of weather on freshwater fis predator-prey dynamics. In: Predutor-prey systems in fisheries munagement. R.H. Stroud y $\mathrm{H}$. Clepper (eds): 47-56. Sport Fishing Institute, Washington D.C., USA.

AGGUS, L.R. \& S.A. LEWIS. 1977. Evironmental conditions and standing crops of fishes in predatorstocking-evaluation reservoirs. Procc. Ann. Conference of the Southern Assoc. Fish und Wildlife Agencies, 30: 131-140.

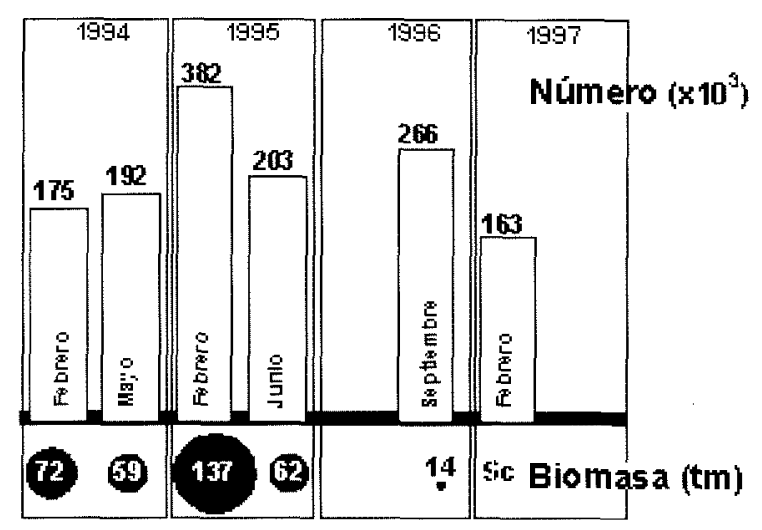

Figura 9. Estimas del número y biomasa de peces a lo largo del estudio. Fish abundance and biomass esteemed from the study. 
DE SILVA, S.S. 1988. ReservoirS of Sri Lanka and theirjisheries. FAO Fish. Tech. Pap. 298, 54 pp.

DUNCAN, A. \& J. KUBECKA. 1995. Land/water ecotone effects in reservoirs on the fish fauna. Hydrobiologia, 303: 11-30.

DOUGLAS, M.E. \& W.J. MATTHEWS. 1992. Does morphology predict ecology?. Hypotheses testing within a freshwater stream fish assemblage. Oikos, 65: 213-224.

FERNANDO, H. \& J. HOLCIK. 1982. The nature of fish communities: a factor influencing the fishery potential and yields of tropical lakes and reservoirs. Hydrobiologia, 97: 127-140.

GRANADO-LORENCIO, C. 1991. Fish communities of spanish reservoir systems: a non-deterministic approach. Verh. Internat. Verein. Limnol., 24: 24282431.

GRANADO-LORENCIO, C. 1996. Ecología de peces. Publicaciones de la Universidad de Sevilla, Sevilla.

GRANADO-LORENCIO, C., E. GUILLÉN y M. CUADRADO. 1985. The influence of some environmental factors on growth of iberian nase, Chondrostoma polylepis, in three reservoirs of western Spain. Cybium, 9(3): 225-232.
GRANADO-LORENCIO， C., L. ENCINA， C. ESCOT, E. MELLADO-ALVAREZ y A. RODRÍGUEZ-RUIZ. 1996. Una nueva metodología para la estima de las poblaciones de peces en embalses. Ingeniería Civil, 3: 35-46.

JENKINS, R.M. 1970. The influence of engineering desing and operation and other environmental factors on reservoirs fisheries. Water Res. Bull. 6: 110119.

KAPETSKY, J.M. \& T. PETR. 1984. Status of African reservoir fisheries. CIFA tech. Paper 10: $46 \mathrm{pp}$.

MERLE, G., D. MOSNIER ET J.N. TOURENQ. 1994. La vidange de la retenue de Pareloup en 1993: une étape-clef dans la vie du lac. Hydroécol. Appl., 6 (1-2): 427-446.

RODRIGUEZ-RUIZ, A. C. GRANADO-LORENCIO. 1992. Spawning period and migration of the three species of cyprinids in a stream with mediterranean regimen (SW Spain). J. Fish Biol., 41 :545-556.

SANCHO, F. y C. GRANADO-LORENCIO. 1988. La pesca en los embalses andaluces. Instituto de Desarrollo Regional, Sevilla, 223 pp. 
